\title{
3D Buoyancy Induced Heat Transfer in Triangular Solar Collector Having a Corrugated Bottom Wall
}

\author{
Walid Aich \\ Mechanical Engineering Department \\ University of Hail, Saudi Arabia \\ Research Unit of Materials, Energy and Renewable Energies (MEER) \\ Faculty of Sciences of Gafsa, Tunisia \\ aich_walid@yahoo.fr
}

\begin{abstract}
A numerical study of the natural convection heat transfer and fluid flow in 3D triangular solar collector having a corrugated bottom wall has been carried out using finite volume method. The aim of the study is to investigate how buoyancy forces influence airflow and temperature patterns inside the collector heated from below and cooled on its inclined walls while vertical ones are assumed to be perfect thermal insulators. Rayleigh number is the main parameter which changes from $10^{3}$ to $10^{5}$ and Prandtl number is fixed at $P r=0.71$. Results are reported in terms of particles trajectories, iso-surfaces of temperature, velocity magnitude and mean Nusselt number. It has been found that the flow structure is sensitive to the value of Rayleigh number and that heat transfer is enhanced with increasing of this parameter.
\end{abstract}

Keywords-natural convection; heat transfer; solar collector; Rayleigh number; Nusselt number

\section{INTRODUCTION}

A prodigious importance has been given to the natural convection heat transfer phenomena which can be seen in many energy-related applications, such as thermal insulation of buildings using air gaps, solar energy collectors, furnaces and fire control in buildings and so on. These applications are reviewed by many authors in the literature [1-4]. The shape of the enclosure is the most effective parameter for natural convection studies in enclosure. The enclosures encountered in these applications are highly diverse in their geometrical configuration and the most investigated enclosures include the annulus between horizontal cylinders, the spherical annulus, the hollow horizontal cylinder, the closed rectangular cavity, and the closed triangular cavity. Pioneers studied natural convection in triangular cavities in [5-7]. Authors in [8,9] modeled the winter and summer day boundary conditions inside a roof of triangular cross-section. Assuming a geometric symmetry through the midplane of an isosceles triangular cavity, author in [10] examined a right angled triangular cavity with a heated bottom wall, cooled hypotenuse, and insulated vertical wall. Various aspect ratios and preselected Rayleigh numbers were used for the reduced right-angled triangular cavity filled with air or water. Solutions of the time-dependent conservation equations were obtained using two different numerical techniques, which while yielding different numerical values for the velocity and temperature fields, did not alter the flow structure pertinent to a single convective cell for low Rayleigh number and to a multicellular regime for high Rayleigh number. Authors in [11] carried out the natural convection problem with flush mounted heater on one wall of a triangular cavity. Governing parameters on heat transfer and flow fields are triangle aspect ratio, heater location, heater length and Rayleigh number. They observed that the most important parameter on heat transfer and flow field is the heater position which can be a control parameter for their system.

Authors in [12] analyzed a numerical study of heat and mass transfer due to natural convection inside a triangular enclosure. The governing equations of the two-dimensional flow problem consist of a velocity-pressure (UVP) formulation along with the energy and concentration flow equations. These equations are solved numerically by control volume based finite element method using the equal-order method without pressure correction. They found that the buoyancy ratio and the Lewis number values have a profound influence on the thermal, concentration and dynamic fields. Steady state laminar natural convection in right triangular and quarter circular enclosures was investigated in [13]. The presented results showed that both aspect ratios and Rayleigh number have a strong influence on the streamline patterns and isotherms. A sizable amount of other related studies can be found in the literature review [1425]. Only limited attention has been paid to the study of threedimensional transverse flow which is primordial when dealing with the enhancement of heat transfer. The paramount aim of this work has been to numerically investigate the diffusive natural convection heat transfer and fluid flow in a threedimensional triangular solar collector heated from its corrugated bottom wall.

\section{MATHEMATICAL FORMULATION}

\section{A. Physical Model}

The considered physical model is presented in Figure 1 with its specified coordinate system and boundary conditions. The model is a triangular shape solar collector with a corrugated bottom wall. The gravity acts in the negative y axis 
and the cavity is formed by the heated corrugated absorber plate, the two cooled inclined glass covers and the two vertical adiabatic walls.

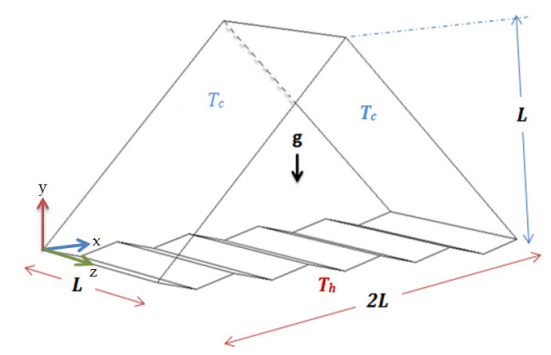

Fig. 1. Schematic of the problem with domain and boundary conditions.

\section{B. Governing Equations and Numerical Solution}

As numerical method we used the vorticity-potential vector formalism $(\vec{\psi}-\vec{\omega})$ which allows, in a three-dimensional configuration, the elimination of the pressure, which is a delicate term to treat. To eliminate this term one applies the rotational to the equation of momentum. More details on this 3$\mathrm{D}$ formalism can be found in [26]. The potential vector and the vorticity are respectively defined by the two following relations:

$$
\vec{\omega}^{\prime}=\vec{\nabla} \times \vec{V}^{\prime} \text { and } \vec{V}^{\prime}=\vec{\nabla} \times \vec{\psi}^{\prime}
$$

After nondimensionalization, the system of equations controlling the phenomenon becomes as:

$$
\begin{aligned}
& -\vec{\omega}=\nabla^{2} \vec{\psi} \\
& \frac{\partial \vec{\omega}}{\partial t}+(\vec{V} \cdot \nabla) \vec{\omega}-(\vec{\omega} \cdot \nabla) \vec{V}=\Delta \vec{\omega}+R a \cdot \operatorname{Pr} \cdot\left[\frac{\partial T}{\partial z} ; 0 ;-\frac{\partial T}{\partial x}\right] \\
& \frac{\partial T}{\partial t}+\vec{V} \cdot \nabla T=\Delta T
\end{aligned}
$$

with: $\operatorname{Pr}=\frac{v}{\alpha}$ and $R a=\frac{g \cdot \beta \cdot \Delta T \cdot L^{3}}{v \cdot \alpha}$

Boundary conditions for considered model are given as follows:

Temperature: $T=1$ at the corrugated absorber, $T=0$ at inclined glass covers and $\frac{\partial T}{\partial z}=0$ on vertical walls (adiabatic).

Velocity: $V_{x}=V_{y}=V_{z}=0$ on all walls. The average Nusselt at the hot corrugated wall is given by:

$$
N u_{a v}=\int_{00}^{2} \int_{0}^{1} \frac{\partial T}{\partial n} d x d z
$$

where $\vec{n}$ is the unit vector normal to the hot corrugated wall.

The mathematical model described above was written in a FORTRAN program. The control volume finite difference method is used to discretize governing equations. The central- difference scheme is used for treating convective terms while the fully implicit procedure is used to discretize the temporal derivatives. The grids are considered uniform in all directions with clustering nodes on boundaries. The successive relaxation iteration scheme is used to solve the resulting non-linear algebraic equations. A computer program written for a regular grid was improved to handle the irregularly shaped computational domain using the blocked-off method as described in [27]. In this technique, the whole region is divided into two active and inactive (blocked-off regions) parts. Thus the surface of inclined step in present analysis is approximated by a series of fine cubic steps. It is obvious that using fine grids in the interface region between active and inactive zones causes to have an approximate boundary which is more similar to the true boundary. According to the blocked-off technique, known values of the dependent variables must be established in all inactive control volumes. If the inactive region represents a stationary solid boundary as in this case, the velocity components in that region must be equal to zero, and a known temperature (isothermal boundaries) must be established in the inactive control volumes. The control volumes, which are inside the active region, are designated as 1 and otherwise they are 0 . The time step $\left(10^{-4}\right)$ and spatial mesh $(81 \times 81 \times 81)$ are utilized to carry out all the numerical tests. The solution is considered acceptable when the following convergence criterion is satisfied for each time step:

$$
\sum_{i}^{1,2,3} \frac{\max \left|\psi_{i}^{n}-\psi_{i}^{n-1}\right|}{\max \left|\psi_{i}^{n}\right|}+\max \left|T_{i}^{n}-T_{i}^{n-1}\right| \leq 10^{-4}
$$

\section{RESULTS AND DISCUSSION}

Particle trajectories for different Rayleigh number values are illustrated in Figure 2. It is noted that Prandtl number is fixed at $\mathrm{Pr}=0.71$ for the whole work and the Rayleigh number which is the main parameter changes from $10^{3}$ to $10^{5}$. As it can be seen, the flow strength is very low for $\mathrm{Ra}=10^{3}$ and two opposite rotating vortexes are formed showing poor convective heat transfer. Flow strength increases with increasing of Rayleigh number. Indeed the fluid takes up heat energy from the bottom heated absorber and becomes lighter to induce a convective current. Lighter fluid goes up and due to the symmetric nature of colder glass covers, the colder portion of fluid follows the line traced by the colder walls. As a consequence, a significant change in convection is observed and strongest flow pattern is achieved for $\mathrm{Ra}=10^{5}$. In Figure 3 shows the effect of varying Rayleigh number on iso-surfaces of temperature inside the enclosure. At $\mathrm{Ra}=10^{3}$, convection is not significant at all and the iso-surfaces of temperature are parallel to each other and are densely distributed near the bottom heated absorber. The parallel nature of iso-surfaces of temperature shows the domination of conductive heat transfer. However, plumelike distribution on temperature field is observed at $\mathrm{Ra}=10^{4}$ due to the starting of convection regime. Iso-surfaces are seen to be densely packed along the boundaries showing that convection is very strong in those regions. At the corners these iso-surfaces are more and more densely packed showing the presence of a strong conduction heat transfer. The temperature distribution assumes a distorted shape near the 
middle and the top due to increasing of effectiveness of convection heat transfer. Strong convection occurred for $\mathrm{Ra}=10^{5}$ and stronger plumelike distribution is observed.

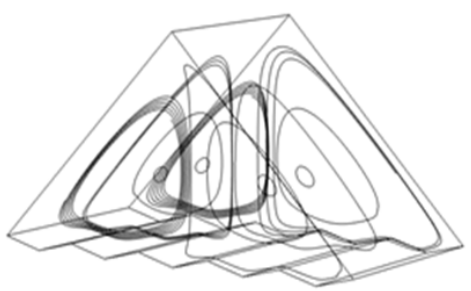

(a)

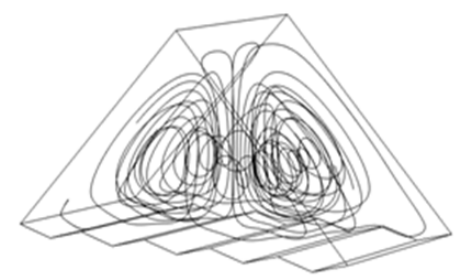

(b)

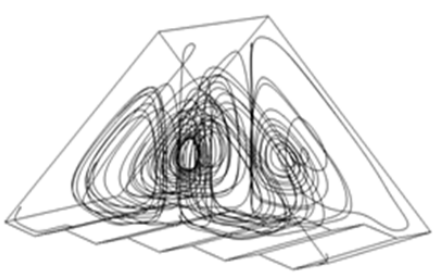

(c)

Fig. 2. Particles trajectory for different Ra values: (a) $10^{3}$, (b) $10^{4}$, (c) $10^{5}$

The velocity magnitude of the fluid flow inside the enclosure is presented in Figure 4 for different Rayleigh number values. At the lowest value of this parameter and due to the domination of conduction regime of heat transfer, the fluid is almost at rest in the enclosure with negligible motion at the middle far from boundaries. By increasing $\mathrm{Ra}$, the heated and rising fluid leads to formation of the thermal boundary layer at the upper part of the inclined walls and there is a strong convective current in the middle of the cavity while corners remain as stagnant zones. This result supports our observations made during the discussion on the particles trajectory and temperature distribution. The highest magnitude of velocity occurred for $\mathrm{Ra}=10^{5}$ and a very strong double plumelike distribution is observed due to increasing of effectiveness of convection heat transfer and fluid flow at the middle of the collector. It is noticed that the heat transfer rate inside the enclosure is measured in terms of the overall Nusselt number. Figure 5 shows the variation of the average Nusselt number with the Rayleigh number Ra. It is obvious that for low values of $\mathrm{Ra}$ and when the conduction is the dominant mode of heat transfer, this variation is insignificant. However, for $\mathrm{Ra}>10^{4}$ heat removal from the heated corrugated absorber increases by means of increasing $\mathrm{Ra}$ and the maximum rate is obtained for the highest $\mathrm{Ra}$ as expected. The corrugated form of the bottom of the collector is found to enhance heat transfer due to increasing of heated surface when compared with simple plane wall.

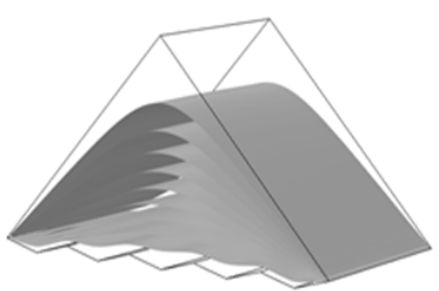

(a)

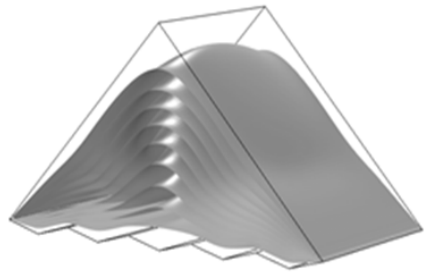

(b)

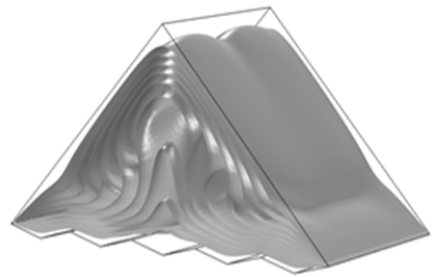

(c)

Fig. 3. Iso-surfaces of temperature for different Ra values: a) $10^{3}$, (b) $10^{4}$, (c) $10^{5}$

\section{CONCLUSION}

Three-dimensional numerical investigation has been carried out to simulate buoyancy induced heat transfer and fluid flow inside a triangular enclosure with corrugated bottom wall. Results are presented for different values of Rayleigh number which is the main parameter of the study. Some of the drawn conclusions are:

- For lower values of Ra conduction is the primary mode of heat transfer and the flow strength is very low due to poor convective heat transfer.

- Flow strength increases with the increasing of $\mathrm{Ra}$ and a strong convective current is noticeable in the middle of the enclosure far from boundaries.

- The flow structure and the magnitude of velocity are sensitive to the value of $\mathrm{Ra}$.

- Overall Nusselt number at the heated surface increases with increasing value of $\mathrm{Ra}$ indicating a maximum heat transfer rate at $\mathrm{Ra}=10^{5}$.

- Even at high Ra the symmetry of the flow is not broken contrary to results of many previous studies in 2D cases.

Further study may include the effect of filling the enclosure with nanoparticles in water based fluid under the effect of magnetic field. 


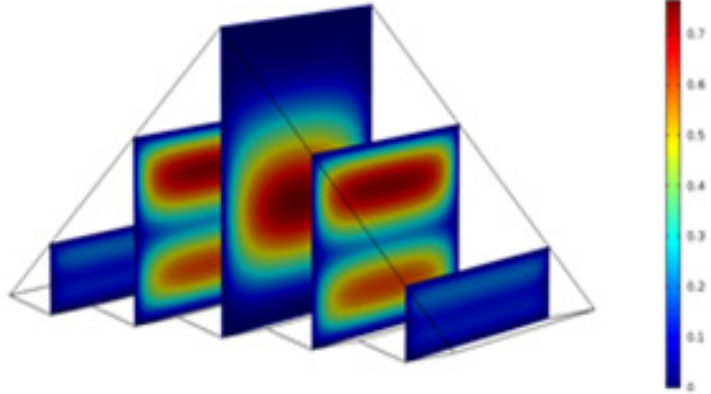

(a)

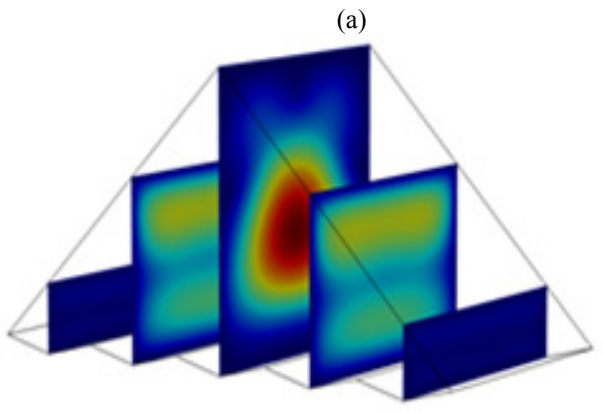

(b)

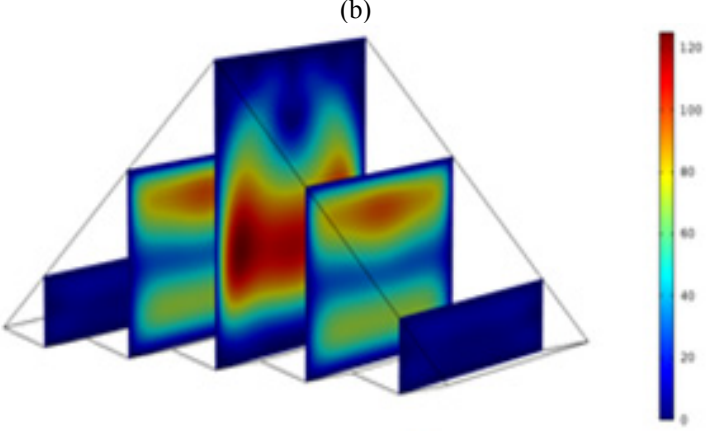

(c)

Fig. 4. Velocity magnitude for different Ra values: a) $10^{3}$, (b) $10^{4}$, (c) $10^{5}$

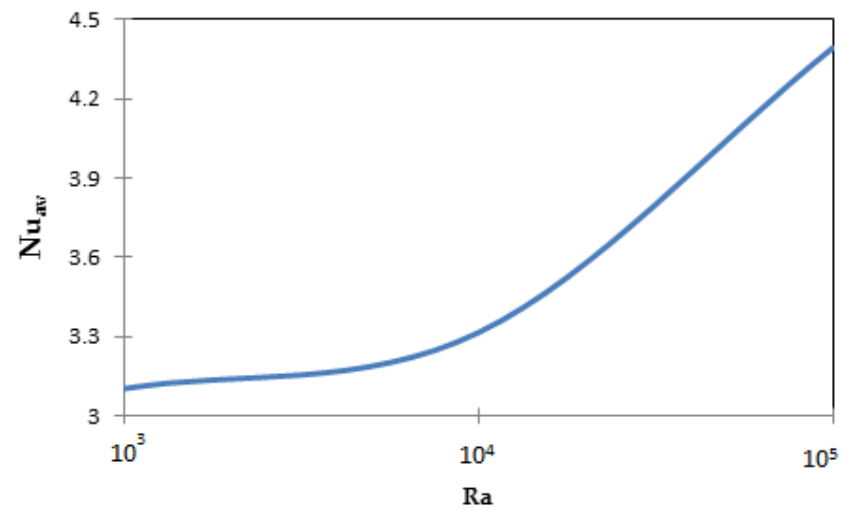

Fig. 5. Variation of mean Nusselt number on hot corrugated wall with Rayleigh number.

\section{ACKNOWLEDGMENT}

Author would like to express his deepest gratitude to Dr. Ali Amri and his institution, "The English Polisher" for their meticulous and painstaking review of the English text of the present paper.

\section{REFERENCES}

[1] G. De Vahl Davis, "Natural convection in a square cavity: a benchmark numerical solution", International Journal for Numerical Methods in Fluids, Vol. 3, No. 3, pp. 249-264, 1983

[2] T. Fusegi, J. M. Hyun, K. Kuwahara, B. Farouk, “A numerical study of three dimensional natural convection in a differentially heated cubical enclosure", International Journal of Heat and Mass Transfer, Vol. 34, No. 6, pp. 1543-1557, 1991

[3] S. C. Tzeng, J. H. Liou, R. Y. Jou, "Numerical simulation-aided parametric analysis of natural convection in a roof of triangular enclosures", Heat Transfer Engineering, Vol. 26, No. 8, pp. 69-79, 2005

[4] A. Omri, J. Orfi, S. Ben Nasrallah, "Natural convection effects in solar stills", Desalination, Vol. 183, No. 1-3, pp. 173-178, 2005

[5] R. D. Flack, "The experimental measurement of natural convection heat transfer in triangular enclosures heated or cooled from bellow", Journal of Heat Transfer, Vol. 102, No. 4, pp. 770-772, 1980

[6] D. Poulikakos, A. Bejan, "Natural convection experiments in triangular enclosure", Journal of Heat Transfer, Vol. 105, No. 3, pp. $652-655,1983$

[7] D. Poulikakos, A. Bejan, "The fluid dynamics of an attic space", Journal of Fluid Mechanics, Vol. 131, pp. 251-269, 1983

[8] H. Asan, L. Namli, "Numerical simulation of buoyant flow in a roof of triangular cross-section under winter day boundary conditions", Energy and Buildings, Vol. 33, No. 7, pp. 753-757, 2001

[9] H. Asan, L. Namli, "Laminar natural convection in a pitched roof of triangular cross-section: summer day boundary conditions", Energy and Buildings, Vol. 33, No. 1, pp. 69-73, 2000

[10] H. Salmun, "Convection patterns in a triangular domain", International Journal of Heat and Mass Transfer, Vol. 38, No. 2, pp. 351-362, 1995

[11] Y. Varol, A. Koca, H. F. Oztop, "Natural convection in a triangle enclosure with flush mounted heater on the wall", International Communications in Heat and Mass Transfer, Vol. 33, No. 8, pp. 951958, 2006

[12] I. Hajri, A. Omri, S. Ben Nasrallah, "A numerical model for the simulation of double-diffusive natural convection in a triangular cavity using equal order and control volume based on the finite element method", Desalination, Vol. 206, No. 1-3, pp. 579-588, 2007

[13] E. Fuad Kent, E. Asmaz, S. Ozerbay, "Laminar natural convection in right triangular enclosures", Heat and Mass Transfer, Vol. 44, pp. 187 200,2007

[14] M. M. Rahman, H. F. Oztop, A. Ahsan, M. A. Kalam, Y. Varol, "Double-diffusive natural convection in a triangular solar collector", International Communications in Heat and Mass Transfer, Vol. 39, No. 2,pp. 264-269, 2012

[15] E. Fuad Kent, "Laminar natural convection in isosceles triangular roofs in wintertime conditions", Heat Transfer Engineering, Vol. 31, No. 13, pp. 1068-1081, 2010

[16] E. Fuad Kent, "Numerical analysis of laminar natural convection in isosceles triangular enclosures", Proceedings of the Institution of Mechanical Engineers, Part C: Journal of Mechanical Engineering Science, Vol. 223, No. 2, pp. 1157-1169, 2009

[17] E. H. Ridouane, A. Campo, "Formation of a pitchfork bifurcation in thermal convection flow inside an isosceles triangular cavity", Physics of Fluids, Vol. 18, No. 7, p. 074102, 2006

[18] E. H. Ridouane, A. Campo, M. Hasnaoui, "Benefits derivable from connecting the bottom and top walls of attic enclosures with insulated vertical side walls", Numerical Heat Transfer, Part A, Vol. 49, No. 2, pp. 175-193, 2006

[19] E. Fuad Kent, "Numerical analysis of laminar natural convection in isosceles triangular enclosures for cold base and hot inclined walls", Mechanics Research Communications, Vol. 36, No. 4, pp. 497-508, 2009 
[20] Y. Varol, H. F. Oztop, I. Pop, "Influence of inclination angle on buoyancy-driven convection in triangular enclosure filled with a fluidsaturated porous medium", Heat and Mass Transfer, Vol. 44, pp. 617624,2008

[21] E. H. Ridouane, A. Campo, M. Mc Garry, "Numerical computation of buoyant airflows confined to attic spaces under opposing hot and cold wall conditions", International Journal of Thermal Sciences, Vol. 44, No. 10, pp. 944-952, 2005

[22] E. H. Ridouane, A. Campo, "Heightened thermal convection as a result of splitting a square cavity diagonally in half", Journal of Electronic Packaging, Vol. 128, No. 3, pp. 251-258, 2006

[23] T. Basak, S. Roy, C. Thirumalesha, "Finite element analysis of natural convection in a triangular enclosure: effects of various thermal boundary conditions", Chemical Engineering Science, Vol. 62, No. 9, pp. 26232640,2007
[24] S. Roy, T. Basak, C. Thirumalesha, C. Murali Krishna, "Finite element simulation on natural convection flow in a triangular enclosure due to uniform and non-uniform bottom heating", Journal of Heat Transfer, Vol. 130, No. 3, p. 032501, 2008

[25] E. H. Ridouane, A. Campo, J. Y. Chang, "Natural convection patterns in right-angled triangular cavities with heated vertical sides and cooled hypotenuses", Journal of Heat Transfer , Vol. 127, No. 10, pp. 11811186,2005

[26] L. Kolsi, A. Abidi, M. N. Borjini, N. Daous, H. Ben Aissia, "Effect of an external magnetic field on the $3-\mathrm{D}$ un steady natural convection in a cubical enclosure", Numerical Heat Transfer, Part A, Vol. 51, No. 10, pp. 1003-1021, 2007

[27] S. V. Patankar, Numerical Heat Transfer and Fluid Flow, CRC Press, 1981 